\title{
Quantitative 3D Assessment of Myocardial Viability with MRI Delayed Contrast Enhancement
}

\author{
V Positano, MF Santarelli, A Pingitore, M Lombardi, L Landini, A Benassi \\ CNR Institute of Clinical Physiology, Pisa, Italy
}

\begin{abstract}
Myocardial viability is a fundamental question in the clinical and therapeutic decision making process. Contrast-enhanced MRI can distinguish between viable and necrotic myocardium in non-invasive manner and with excellent definition of endocardial and epicardial borders. Aim of this study is to propose a software methodology that allows to assess the global, transmural and intramural extent of myocardial necrosis providing both bull-eyes and $3 D$ representation of contrast delayed enhanced area in MRI cardiac images.
\end{abstract}

\section{Introduction}

Myocardial viability is a fundamental question in the clinical and therapeutic decision making process. The extent of viability correlates with recovery of contractile function after revascularization and thereby has relevant prognostic impact in patients with left ventricular dysfunction and coronary artery disease. Contrastenhanced MR (CMR) can distinguish between viable and necrotic myocardium in non-invasive manner and with excellent definition of endocardial and epicardial borders, allowing to assess transmural extent of myocardial necrosis.

Specifically, necrotic myocardium is identified as a hyperenhanced area (HE) at the delayed acquired images after gadolinium administration. Clinical studies showed the accuracy of HE to identify the presence, location and transmural extent of myocardial necrosis [1,2,3]. Assessment of the necrotic area is usually done by visual inspection in qualitative manner. The development of software tools for automatic or semi-automatic assessment of contrast enhanced MR images should provide quantitative data about myocardial necrosis extension and distribution. The use of software tools should also reduce the inter- and intra-observer variability of the contrast enhanced MR images analysis.

\section{MR acquisition protocol}

MRI acquisition was performed using a $1.5 \mathrm{~T}$ whole body MR scanner (GE, CVi - Milwaukee, USA) equipped with high performance gradient (40-mT/m amplitude, 150-mt ms-1 slew rate) and a multichannel receiver with a maximal bandwidth of $250 \mathrm{kHz}$. A 4 element ( 2 anterior and 2 posterior) cardiac phased-array receiver surface coil was used for signal reception. Shortaxis images acquired 20 minutes after bolus injection of $0.2 \mathrm{mmol}$ gadolinium (Gd-DTPA) in end-diastole were used to evaluate myocardial distribution of hyperenhancement. An inversion recovery fast GE sequence was used with the following parameters: echo time, $3.2 \mathrm{sec}$; slice thickness: $8 \mathrm{~mm}$.; spatial resolution, 1.4x1.4 mm.; flip angle, 20 degrees; NEX, 2.

\section{Myocardial viability assessment}

CMR images in DICOM format were transferred to dedicated workstation and processed by a dedicated software tool developed in IDL 5.4 environment. The realized software tool allows semi-automatic detection of LV boundaries and of HE areas. The extent of HE can be evaluated on several slices acquired in short axis direction covering the left ventricle from basal to apex. To assess the apex, one vertical and one horizontal longaxis view are also processed.

The proposed methodology can be summarized as follows:

1. Semi-automatic definition of LV boundaries (i.e. endocardial and epicardial borders) along all acquired slices.

2. Automatic definition of $\mathrm{HE}$ area in the myocardium (as defined in the step 1) in each acquired slice.

3. Automatic definition of anatomical markers (i.e. the anterior and posterior septal insertion of the right ventricle) on all slices.

4. Automatic splitting of myocardium in number of radial sectors defined by the user, starting from the marker(s) defined in the step 3. Sectors can be optionally divided into the inner and outer half (i.e. subendocardial and subepicardial layer).

5. Automatic evaluation of the extent of $\mathrm{HE}$ area defined in the step 2 for each region defined in the step 4. HE area is evaluated as volume $\left(\mathrm{cm}^{3}\right)$ and as percentage of $\mathrm{HE}$ volume respect to myocardial one.

The analysis of the two long-axis views proceeds instead as follows: 
1. Manual definition of apex region in both images.

2. Automatic definition of HE area in the apex region (as defined in the step 1).

3. Automatic evaluation of the extent of $\mathrm{HE}$ area defined in the step 2 for the two apex regions. HE area is evaluated as volume $\left(\mathrm{cm}^{3}\right)$ and as percentage of HE volume respect to myocardial one.

When all needed image are examined, visualization of results as bull-eye representation and $3 \mathrm{D}$ reconstruction is showed. Data about the regional and global extent of HE are also provided.

Figure 1 shows the main phases of the proposed methodology for 4 short axis images: original data are showed in (a). In (b) the result of the semi automatic definition of endocardial and epicardial border is depicted. The automatic definition of HE area is showed in (c). In (d) the myocardium is automatically segmented in six regions.

\subsection{Definition of $L V$ boundaries}

The inner and outer boundaries of the LV were defined on each short axis image as follow. First, a automatic method based on anisotropic filtering and active contour algorithm [4,5] was used to locate preliminary boundaries. The automatic methodology is based on anisotropic filtering of CMR images, able to reduce the amount of noise preserving at the same time detailed structures and sharpening organ boundary edges definition. In fact, in order to preserve edges, the smoothing operation is reduced or even blocked when a discontinuity in the grey image levels is reached. In order to detect the LV boundaries, a deformable model based on Gradient Vector Flow snake (GVF-snake), as a curve that moves through the spatial domain of the image, is applied on the anisotropic filtered images. Snakes or active contours are curves defined within an image domain, which can move under the influence of internal forces coming from within the curve itself, and external forces computed from the image data. The internal and external forces are defined so that the snake will conform to an object boundary or other desired features within an image. In the present application, the snake modelling procedure can start from a rough contour manually traced of internal cavity, then it stabilizes at the firstly local minimum of image energy, corresponding to endocardial border. At this stage, snake model re-starts using previously detected endocardial curve; the algorithm now searches for the first local minimum that is expected to correspond to epicardial surface. The obtained boundaries was examined by the operator and eventually corrected on each image.

\subsection{Automatic definition of HE area}

The HE regions in myocardium were automatically extracted by fuzzy c-mean (FCM) clustering method that automatically classifies the myocardium in necrotic and normal regions. The FCM approach [6,7] is able to do unsupervised classification of data in a number of clusters, identifying different tissue in an image without the use of an explicit threshold. In our approach, pixel values related to myocardial region are segmented in two clusters (i.e. HE and basal). The user can review and correct the computer generated results.

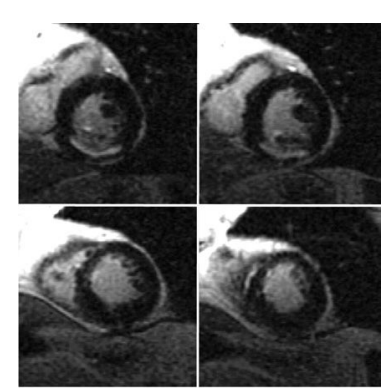

a

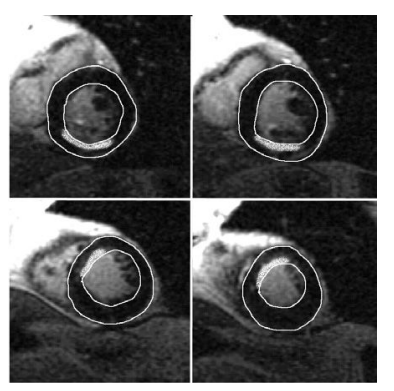

c

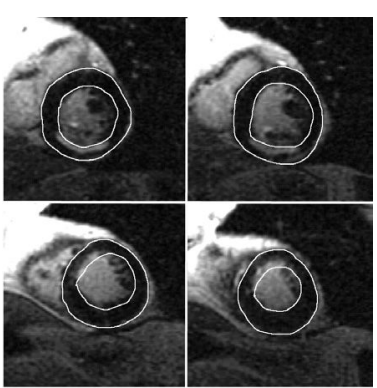

$\mathrm{b}$

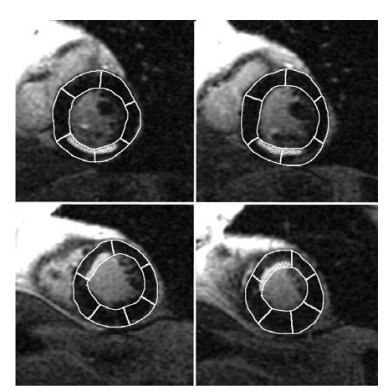

d
Figure 1: Method phases: original images (a), myocardial contour detection (b), hyper-enhanced area detection(c), myocardium segmentation (d).

The fuzzy C-means algorithm performs a classification of image data by computing a measure of membership, called the fuzzy membership, at each pixel for a specified number of classes. The fuzzy membership function, constrained to be between zero and one, reflects the degree of similarity between the image pixel at that location and the prototypical data value or centroid of its class. Thus, a membership value near unity means that the image pixel is close to the centroid for that particular class. FCM is formulated as the minimization of the following objective function with respect to the membership function $\mathrm{u}$ and centroids $\mathrm{v}$ :

$$
J_{F C M}=\sum_{j \in \Omega} \sum_{k=1}^{C} u_{j k}^{q}\left\|y_{j}-v_{k}\right\|^{2}
$$

where $\Omega$ represents the pixel location in image domain, $\mathrm{q}$ is a parameter greater that one that determines 
the amount of fuzziness of the classification $(q=2$ in our application), $u_{j k}$ is the membership value at location $\mathrm{j}$ for class $\mathrm{k}, y_{j k}$ is the intensity value at $\mathrm{j}$ location, $v_{k}$ is the centroid of the class $\mathrm{k}, \mathrm{C}$ is the number of classes $(\mathrm{C}=2$ in our problem).

When the above objective function is minimized, the value of $u_{j k}$ is approaching to one only if the pixel intensity at $\mathrm{j}$ location is close to the centroids of class $\mathrm{k}$. Similarly, the value of $u_{j k}$ is approaching to zero only if the pixel intensity at $\mathrm{j}$ location is far from the centroids of class $\mathrm{k}$. Also, the pixels with same intensity value would be grouped into same groups with same probability.

Minimization of $J_{F C M}$ is based on the suitable selection of $\mathrm{u}$ and $\mathrm{v}$ using an interactive process through the following equations:

$$
\begin{aligned}
& u_{j k}=\left(\sum_{i=1}^{C}\left(\frac{\left\|y_{j}-v_{k}\right\|^{2}}{\left\|y_{i}-v_{k}\right\|^{2}}\right)^{\frac{2}{q-1}}\right)^{-1} \\
& v_{i}=\frac{\sum_{j \in \Omega} u_{j k}^{q} y_{j}}{\sum_{j \in \Omega} u_{j k}^{q}}
\end{aligned}
$$

The algorithm stops when the value of $u_{j k}$ will converge.

\subsection{Anatomical markers}

The user has to define on or two reference points (i.e. the anterior and optionally posterior septal insertion of the right ventricle). The program attempts to automatic define markers looking for the septal insertion of the right ventricle with the left one, the obtained point can be corrected by the operator.

\subsection{Automatic splitting of myocardium}

The myocardium is automatically divided into a defined number of equiangular sectors starting from the reference point. Optionally, the myocardium can be divided into the inner and outer half (i.e. subendocardial and subepicardial layer). In this way, in each slice the left ventricle wall can be automatically divided in a number of regions, ranging from 3 to 24 .

\subsection{Evaluation of the extent of $\mathrm{HE}$ area}

The myocardial mass related to each sector is evaluated, tacking into account the geometrical information extracted from the DICOM files and a value of $1.05 \mathrm{~g} / \mathrm{ml}$ for the density of the myocardial tissue. The percentage of $\mathrm{HE}$ area respect to the myocardium mass was also automatically evaluated. For each sector, the method provides the values of myocardial mass and the necrotic mass. Data coming from all sectors can be joined to provide the total value of necrotic mass in the LV and the total percentage of necrotic defect.

Each sector can also classified in order to provide a score for the necrosis severity, an in order to classify the necrotic lesion in transmural or intramural. The classification is done following the

\subsection{Results visualization}

Both numeric and bull-eye representation of the 3D data volume are provided. In particular, the program calculate the global extent of the necrotic area, the extent of necrotic area respect to the myocardial mass. The value of $\mathrm{HE}$ extension for each region can be visualized by a bull-eye representation, in which each value of HE extension corresponds to a color in a suitable color map. The Figure 2.a provides an example of the bull-eye representation, in which 7 short axis slices were acquired and 6 radial sectors and two anatomical markers were defined.
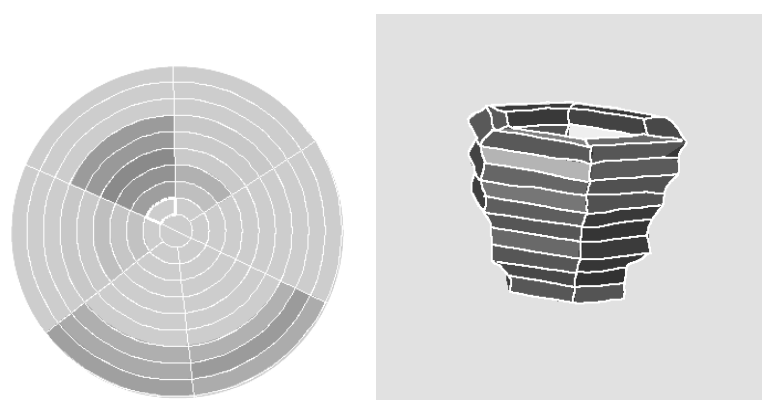

Figure 2: bull-eye and $3 D$ representation of hiperenhanced area distribution.

Moreover, from evaluating the $\mathrm{HE}$ percentage parameter for each region relevant to each slice covering all the heart, it is possible to graphically visualize all resulting data as a 3D parametric map. Then, by exploiting graphical tools, it is possible to process data in a 3D fashion for a better determination of the entity and extension of the necrotic region.

\section{Result and conclusions}

Contrast delayed enhancement can be considered a noninvasive method of magnetic resonance to evaluate directly the presence and extent of myocardial necrosis in human. In particular, the transmural extent is inversely correlated to the early restoration of flow, mimicking the wavefront phenomenon of the transmural extension of infarction, and is an important predictor of future 
improvement in contractile function.

Quantitative analysis of CMR data requires a robust and fast methodology, able to assess the extent of $\mathrm{HE}$ area in effective manner. The main requirements in developing such methodology are a strong reduction of the required processing time, the method robustness respect to the operator and the ability to provide a correct evaluation of myocardial viability.

About the first point, we show that the use of automatic or semi-automatic approach allows to reduce the required processing time to about five minutes, a time compatible with the clinical practice. Moreover, the operator independence of the methodology assures a good robustness of the proposed approach.

\section{References}

[1] Wu E, Judd RM, Vargas JD, Klocke FJ, Bonow RO, Kim RJ. Visualization of presence, location, and transmural extent of healed Q-wave and non-Q-wave myocardial infarctio. Lancet 2001; 357:21-28

[2] Ramani K, Judd RM, Holly TA, Parrish TB, Rigolin VH, Parker MA, Callahan C, Fitzgerald SW, Bonow RO, Klocke FJ. Contrast magnetic resonance imaging in the assessment of myocardial viability in patients with stable coronary artery disease and left ventricular dysfunction. Circulation 1998; 98:2687-2694
[3] Kitagawa K, Sakuma H, Hirano T, Okamoto S, Makino K, Takeda K. Acute Myocardial Infarction: Myocardial Viability Assessment in Patients Early ThereafterComparison of Contrast-enhanced MR Imaging with Resting ${ }^{201}$ Tl SPECT ${ }^{1}$, Radiology 2003;226:138-144.

[4] Santarelli MF, Positano V, Michelassi C, Lombardi M, Landini L. Automated cardiac MR image segmentation: theory and measurement evaluation. Medical Engineering and Physics, 2003;25:2:149-159.

[5] Positano V, Santarelli MF, Landini L. Automatic Characterization of Myocardial Perfusion in Contrast Enhanced MRI. EURASIP Journal on Applied Signal Processing 2003;5:413-422.

[6] Udupa JK, Samarasekera S. Fuzzy connectedness and object definition: Theory, algorithm and application in image segmentation. Graphical Models Image Processing. 1996:58: 3:246-261.

[7] Bezdek J, Hall L, Clarke L, Rewiew of MR image segmentation using pattern recognition. Med Phys.,1993; 20:1033-1948.

Address for correspondence.

Vincenzo Positano

CNR Institute of Clinical Physiology

CNR Research Area - Via G. Moruzzi 1

56124 PISA (ITALY)

E-mail address: positano@ifc.cnr.it 